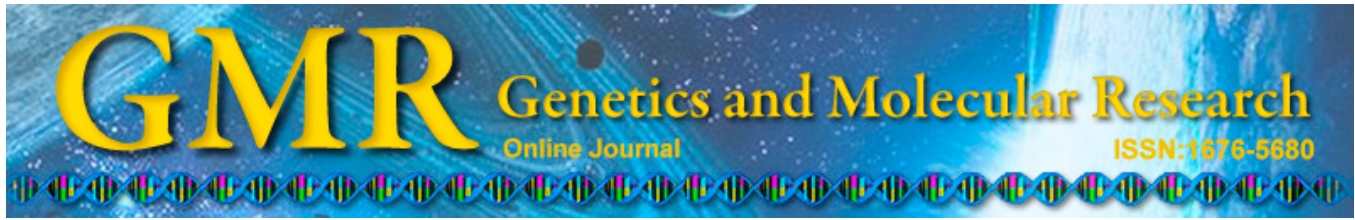

\title{
Amiloride, a urokinase-type plasminogen activator receptor (UTPA) inhibitor, reduces proteinurea in podocytes
}

\author{
L.B. $\mathrm{Xu}^{1}$, N. Chi ${ }^{2}$ and W. Shi ${ }^{1}$ \\ ${ }^{1}$ Department of Nephrology, Guangdong General Hospital of Southern \\ Medical University, Guangzhou, China \\ ${ }^{2}$ Department of Nephrology, Zhujiang Hospital of Southern Medical University, \\ Guangzhou, China \\ Corresponding author: W. Shi \\ E-mails: shiweidn@126.com / shiwei729@yeah.net
}

Genet. Mol. Res. 14 (3): 9518-9529 (2015)

Received November 17, 2014

Accepted March 23, 2015

Published August 14, 2015

DOI http://dx.doi.org/10.4238/2015.August.14.15

\begin{abstract}
This study examined the mechanism of action of amiloride, a urokinase-type plasminogen activator receptor inhibitor, in lowering proteinuria. Podocytes were resuscitated to allow for their proliferation and were observed for morphological changes. In the in vitro experiment, control, lipopolysaccharide, and lipopolysaccharide + amiloride groups were established. The expression of urokinasetype plasminogen activator receptor (UPAR) in podocytes was detected with a flow cytometer and cell motility was detected with the transwell migration assay. In the in vivo test, the urine protein volume of the model was detected at $24 \mathrm{~h}$ using Coomassie brilliant blue staining and the morphological changes of the podocytes were detected with immunofluorescence. The protein expression rate of uPAR in the lipopolysaccharide group was significantly higher than those in the control and lipopolysaccharide + amiloride groups $(\mathrm{P}<0.05)$. The viability of cells in the lipopolysaccharide group was significantly
\end{abstract}


higher than those in the control and lipopolysaccharide + amiloride groups $(\mathrm{P}<0.05)$. Compared with the urine protein level in the control group at $24 \mathrm{~h}$, the level in the lipopolysaccharide group increased significantly $(\mathrm{P}<0.05)$, whereas compared with the urine protein level in the lipopolysaccharide group, the level in the lipopolysaccharide + amiloride group decreased $(\mathrm{P}<0.05)$. uPAR expression was significantly downregulated, and the fusion of the podocyte-specific skelemin synaptopodin on the glomerulus podocytes was significantly decreased in the lipopolysaccharide + amiloride group. These results suggest that amiloride is able to reduce cell motility and thus lower proteinuria by inhibiting the expression of UPAR in podocytes.

Key words: Urokinase-type plasminogen activator receptor; Inhibitor; Proteinuria; Amiloride; Nephropathy

\section{INTRODUCTION}

Proteinuria is one of the most common clinical manifestations in renal diseases and is also an important factor associated with the continuous progression of renal diseases. A series of large-scale clinical studies have demonstrated that the volume and duration of proteinuria have a direct bearing on the prognosis of renal diseases (Hasegawa et al., 2013). Podocytes are inherent cells of the glomerulus and adhere to the outside of the basilar membrane therein, constituting a filtration barrier along with the basilar membrane itself and the endothelial cells of blood capillaries (Lasagni and Romagnani, 2013; Müller-Krebs et al., 2013). Therefore, as an important constituent of the glomerulus filtration membrane, podocytes play a critical role in the progression of proteinuria-related renal diseases, and podocyte dysfunction or damage might lead to the occurrence of proteinuria (Li et al., 2013; Reiser, 2013). Current research has found that increased expression of the urokinase-type plasminogen activator receptor (uPAR) might lead to enhanced podocyte activity and the generation of proteinuria in diseased human glomeruli, nephropathy in animal models, and proteinuria in animal and cell models induced by lipopolysaccharides (Yu et al., 2013a).

Amiloride is a Na channel blocker that has been used clinically as a diuretic. Research has indicated that amiloride is able to inhibit the expression of uPAR, thus reducing cell motility in cloned tumor cell strains at mRNA and protein levels (Zhang et al., 2012). The question arises, therefore, of whether amiloride might be able to inhibit the expression of uPAR and thereby reduce the mobility of podocytes. In this study, models of lipopolysaccharide-induced transient proteinuria in mice and of lipopolysaccharide-induced podocyte damage in cell culture were established to observe the effects of uPAR expression in vivo and in vitro, respectively, to preliminarily address the mechanism of action of amiloride in lowering proteinuria, and to provide a new therapeutic paradigm for the treatment of proteinuria at the level of target podocytes.

\section{MATERIAL AND METHODS}

\section{Material}

Immortal mouse podocytes were purchased from the Shanghai Fusheng Biotechno- 
logical Development Co., Ltd. (Shanghai, China) and cryopreserved in liquid nitrogen for later use. We purchased 30 C57BL/6 male mice weighing 20-25 g from the Shanghai Laboratory Animal Centre of The Chinese Academy of Sciences for these studies.

Goat polyclonal and goat anti-mouse antibodies were purchased from Shanghai Shrek Biotechnology Co., Ltd. (Shanghai, China). FITC labeled rabbit anti-goat IgG was purchased from Amresco Co. (Solon, OH, USA); RPMl640 and low-glucose Dulbecco's modified Eagle medium (DMEM) culture media were purchased from Beijing Kang Pu Hui Wei Technology Co., Ltd. (Beijing, China); fetal bovine serum (FBS) was purchased from the Shanghai Baoman Biotechnology Co. Ltd. (Shanghai, China); Coomassie brilliant blue was purchased from Beijing Bole Bioscience Development Co. Ltd. (Beijing, China); the RNAse-Free DNA enzyme kit was purchased from Invitrogen (Carlsbad, CA, USA); lipopolysaccharides were purchased from Shanghai Hualan Chemical Technology Co., Ltd. (Shanghai, China); and amiloride (batch No. 20130518) was purchased from Hubei Zhongcheng Pharmaceutical Co., Ltd. (Wuhan, China).

BM-37XB immunofluorescence inverted phase contrast microscope (Hach Co., Loveland, $\mathrm{CO}$, USA); DELTA ordinary optical microscope (API, USA); the $\mathrm{CO}_{2}$ incubator was purchased from LEAD-TECH Scientific Instrument Co., Ltd. (Shanghai, China).

\section{Resuscitation and proliferation of podocytes}

A $75-\mathrm{cm}^{2}$ culture flask was washed 3 times with type-I collagen coating PBS for later use. The mouse podocytes cryopreserved in liquid nitrogen were thawed rapidly (within $1 \mathrm{~min}$ ) in a water bath preheated to $37^{\circ} \mathrm{C}$, and centrifuged at $1500 \mathrm{rpm}$ for $3 \mathrm{~min}$. The supernatant was removed and the cells at the bottom were resuspended in a new centrifuge tube with $1.5 \mathrm{~mL}$ FBS with pipetting. The uniformly pipetted cell suspension was added to the $75-\mathrm{cm}^{2}$ culture flask, to which $12.5 \mathrm{~mL}$ RPMI 1640 culture solution containing 20-100 U/mL interferon (IFN)- $\gamma$ had previously been added. The flask was shaken slightly, and cultured in an incubator containing $5 \% \mathrm{CO}_{2}$ for proliferation at $33^{\circ} \mathrm{C}$ with the medium replaced every 2 days.

\section{Morphological observation and identification of podocytes}

The podocytes that had differentiated at 14 days after being cultured at $33^{\circ} \mathrm{C}$ in a solution containing IFN- $\gamma$ or at $37^{\circ} \mathrm{C}$ in a solution containing no IFN- $\gamma 14$ were photographed microscopically and observed for any morphological changes. The podocytes cultured at $37^{\circ} \mathrm{C}$ for 14 days were identified as maturely differentiated podocytes using anti-synaptopodin podocyte specific protein immunofluorescence (Shi et al., 2013).

\section{In vitro experimental groups and interventional treatment of podocytes}

The control group consisted of podocytes without any intervention factor. The lipopolysaccharide group was generated by incubation of $50 \mathrm{mg} / \mathrm{L}$ lipopolysaccharide with maturely differentiated podocytes for $24 \mathrm{~h}$. The lipopolysaccharide + amiloride group was generated with addition of both $50 \mathrm{mg} / \mathrm{L}$ lipopolysaccharide and $50 \mathrm{mg} / \mathrm{L}$ amiloride to the mature podocytes for $24 \mathrm{~h}$. 


\section{Detection of uPAR expression in podocytes using flow cytometry}

Cells were dissociated with the dissociation buffer and the number of podocytes was adjusted to $1 \times 10^{9} / \mathrm{L}$. The podocytes were centrifuged at $1000 \mathrm{rpm}$ for $5 \mathrm{~min}$ and the supernatant was discarded. The podocytes were washed 3 times with phosphate buffered saline (PBS), incubated with $0.5 \mu \mathrm{g}$ primary antibody goat anti-mouse uPAR (AF-534) for $20 \mathrm{~min}$, centrifuged at $1000 \mathrm{rpm}$ for 5 min after addition of $1000 \mu \mathrm{L}$ cold PBS, incubated with $0.4 \mu \mathrm{g}$ FITC marked rabbit anti-goat IgG for $20 \mathrm{~min}$ in the dark after the supernatant was discarded, and centrifuged at $1000 \mathrm{rpm}$ for $5 \mathrm{~min}$ after addition of $1000 \mu \mathrm{L}$ cold PBS. Refer to the previous experiment, the supernatant was discarded and the cells were re-suspended in $200 \mu \mathrm{L}$ PBS, mixed well, and placed in a flow cytometric test tube to detect the expression of the uPAR protein in the podocytes (Wei et al., 2008).

\section{Detection of podocyte motility with the transwell migration assay}

An I-type collagen coated cell culture insertion transwell plate was used for this assay. The plate was stored at room temperature for $1 \mathrm{~h}$ and was washed once with PBS. Fifteen milliliters fresh DMEM culture medium containing no IFN- $\gamma$ and a final concentration of $10 \%$ FBS in transwell plate. The podocytes that had been cultured at $37^{\circ} \mathrm{C}$ and maturely differentiated, and treated on the basis of their respective experimental groups, were inoculated onto the transwell plate at $1 \times 10^{4} / \mathrm{mL}$ (final concentration), and incubated for $24 \mathrm{~h}$ in an incubator containing $5 \% \mathrm{CO}_{2}$ at $37^{\circ} \mathrm{C}$. The cells that had not migrated and remained on the upper layer of the membrane were removed gently with a swab. The migrated cells on the lower layer were fixed for 10-20 min with 4\% paraformaldehyde, washed twice with PBS, stained for 5 min with $0.1 \%$ gentian violet prepared with $20 \%$ ethyl alcohol, and washed again for 1-3 min with PBS. The filter membrane was cut, horizontally placed on a slide, and photographed under the phase contrast microscope in the central field, and the migrated cells were counted (Ilatovskaya et al., 2013).

\section{Establishment of animal models}

Thirty C57BL/6 male mice were randomly divided into control, lipopolysaccharide treatment (lipopolysaccharide), and lipopolysaccharide + amiloride treatment (lipopolysaccharide + amiloride) groups, with 10 animals in each group. The amiloride was dissolved in PBS and stored at $-20^{\circ} \mathrm{C}$; the lipopolysaccharide was dissolved in normal saline and stored at $4^{\circ} \mathrm{C}$. The lipopolysaccharide and lipopolysaccharide + amiloride groups were intraperitoneally injected with $200 \mu \mathrm{g}$ lipopolysaccharide. The control group was intraperitoneally injected with an equivalent amount of saline. The lipopolysaccharide + amiloride group was intragastrically administered $10 \mathrm{mg} \cdot \mathrm{kg}^{-1} \cdot \mathrm{d}^{-1}$ of amiloride 2 days ahead of the lipopolysaccharide injection. The control and lipopolysaccharide groups were intragastrically administered an equivalent amount of normal saline. The urine protein collected at $24 \mathrm{~h}$ was retained and tested on the second day; harvesting of tissues on Day 3, and each mouse was anesthetized with ether and routinely sterilized. The mouse abdominal cavity was cut open to draw a blood sample from the abdominal aorta. The blood was centrifuged and the supernatant was taken. The tissue of the two kidneys was taken and divided into four parts: one part was fixed with $4 \%$ paraformaldehyde, one was fixed with $2.5 \%$ glutaraldehyde, one was cryopreserved in liquid 
nitrogen, and the fresh cortex of the remaining part was taken and delivered to the pathology department to generate sample slides after being rapidly sectioned with a freezing microtome (Abe et al., 2013).

\section{Coomassie brilliant blue detection of the urine protein volume in the model within $24 \mathrm{~h}$}

Urine $(0.1 \mathrm{~mL})$ and $5 \mathrm{~mL}$ Coomassie brilliant blue reagent solution were added to the test tube and $0.1 \mathrm{~mL}$ normal saline and $5 \mathrm{~mL}$ Coomassie brilliant blue reagent solution were added to the blank control tube; the tube contents were well mixed. A colorimetric assay was performed after 5-10 min at $595 \mathrm{~nm}$ using a spectrophotometer. The numerical value was read and the standard curve was consulted to determine protein concentration.

To dilute the standard protein solution and generate a $1 \mathrm{mg} / \mathrm{mL}$ standard protein solution, $1 \mathrm{~mL} 5 \%$ stock protein solution $+49 \mathrm{~mL}$ normal saline were added. Diluted solution $(0.1$ $\mathrm{mL}$ ) was taken from each tube and well mixed with $5 \mathrm{~mL}$ Coomassie brilliant blue reagent solution for colorimetric assessment after $5 \mathrm{~min}$. The colorimetric tube was washed with $95 \%$ ethyl and then washed with pure water after each tube was tested (Sanchez-Niño et al., 2013).

\section{Immunofluorescence detection of the podocytes}

Fresh mouse renal tissue was immediately processed into $3 \mu \mathrm{m}$ thick frozen sections, fixed for $10 \mathrm{~min}$ at $-20^{\circ} \mathrm{C}$ with acetone, washed 3 times with PBS for 5 min each time, permeated with $0.5 \%$ Triton X-100 for 20 min, washed an additional 3 times with PBS for 5 min each time, and blocked in 5\% bovine serum albumin (BSA) for $20 \mathrm{~min}$ at room temperature. The slides were placed in a humidified box after addition of the primary antibody and incubated at $4^{\circ} \mathrm{C}$ overnight, washed 3 times with PBS for 5 min each time, incubated at $37^{\circ} \mathrm{C}$ for $60 \mathrm{~min}$ in the dark after addition of the fluorescent labeled secondary antibody, and washed 3 times with PBS for 5 min each time. The slides were subsequently mounted with an antifluorescence attenuation reagent, and observed under a fluorescence microscope.

\section{Statistical analysis}

The measurement data are reported as means \pm standard deviation. The SPSS 13.0 statistical software (SPSS, Chicago, IL, USA) was used for statistical analysis. The method of one-way analysis of variance was used for the results. The homogeneity of variance was subject to least significant difference (LSD) multiple comparisons among groups and the heterogeneity of variance was subjected to Dunnett's T3 multiple comparisons among groups. P $<0.05$ was considered to be statistically significant.

\section{RESULTS}

\section{Morphological observation and identification of podocytes}

Podocytes were cultured at $33^{\circ} \mathrm{C}$ in a culture medium containing IFN- $\gamma$. The podocytes were considered to be proliferating if they were alternately arranged like cobblestones or branches. The podocytes were cultured without IFN- $\gamma$ for 14 days at $37^{\circ} \mathrm{C}$ subsequently. As the podocytes became maturely differentiated, the cell bodies became large, the cytoplasm thinned, and 
many primary and secondary foot processes were intertwined. These features indicated that the podocytes were differentiating. The podocytes that were proliferating after being cultured with IFN- $\gamma$ at $33^{\circ} \mathrm{C}$ did not express the podocyte-specific skelemin synaptopodin, whereas podocytes that had become maturely differentiated after being cultured without IFN- $\gamma$ for 14 days at $37^{\circ} \mathrm{C}$ expressed synaptopodin. In addition, a filose cytoskeleton was observed in all cells (Figure 1).
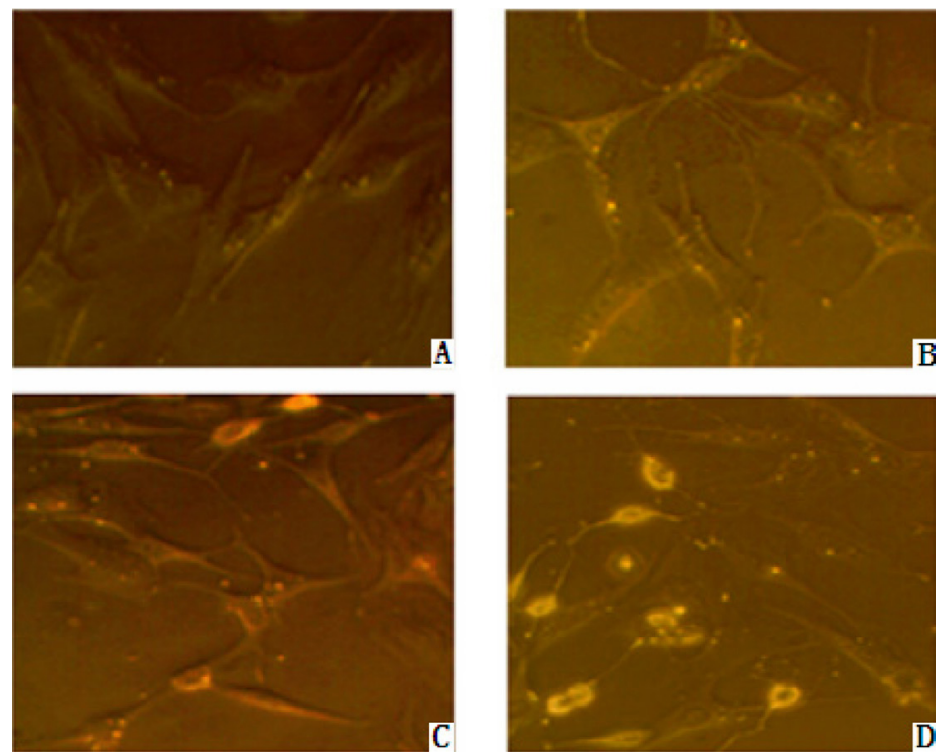

Figure 1. Observation of podocytes cultured in vitro. A) Morphology of the cells cultured for 14 days in a culture medium containing IFN- $\gamma$ at $33^{\circ} \mathrm{C}$; B) podocytes cultured in a culture medium containing IFN- $\gamma$ at $33^{\circ} \mathrm{C}$ did not express the specific skelemin synaptopodin; C) morphology of the cells cultured for 14 days in a culture medium containing no IFN- $\gamma$ at $37^{\circ} \mathrm{C}$; D) Mature podocytes cultured in a culture medium containing no IFN- $\gamma$ at $37^{\circ} \mathrm{C}$ expressed the specific skelemin synaptopodin.

\section{Effect of amiloride on expression of the uPAR protein in podocytes}

The expression of the UPAR protein in the three experimental groups was detected with flow cytometry and the uPAR protein expression rates in the control, lipopolysaccharide, and lipopolysaccharide + amiloride groups were $21.08 \pm 2.17,67.26 \pm 3.59$, and $33.68 \pm$ $2.85 \%$, respectively. The uPAR protein rate in the lipopolysaccharide group was significantly higher than those in the control and the lipopolysaccharide + amiloride groups $(\mathrm{P}<0.05)$ (Table 1 and Figure 2).

Table 1. Flow cytometric detection of uPAR expression changes in different podocytes.

\begin{tabular}{lccc}
\hline Group & Control & Lipopolysaccharide & Lipopolysaccharide + Amiloride \\
\hline uPAR $(\%)($ means \pm SD) & $21.08 \pm 2.17$ & $67.26 \pm 3.59^{*}$ & $33.68 \pm 2.85^{*}$ \\
\hline
\end{tabular}

${ }^{\#}$ Compared with the control group, $\mathrm{P}<0.05$; *compared with the lipopolysaccharide group, $\mathrm{P}<0.05$. uPAR $=$ urokinase-type plasminogen activator receptor. 


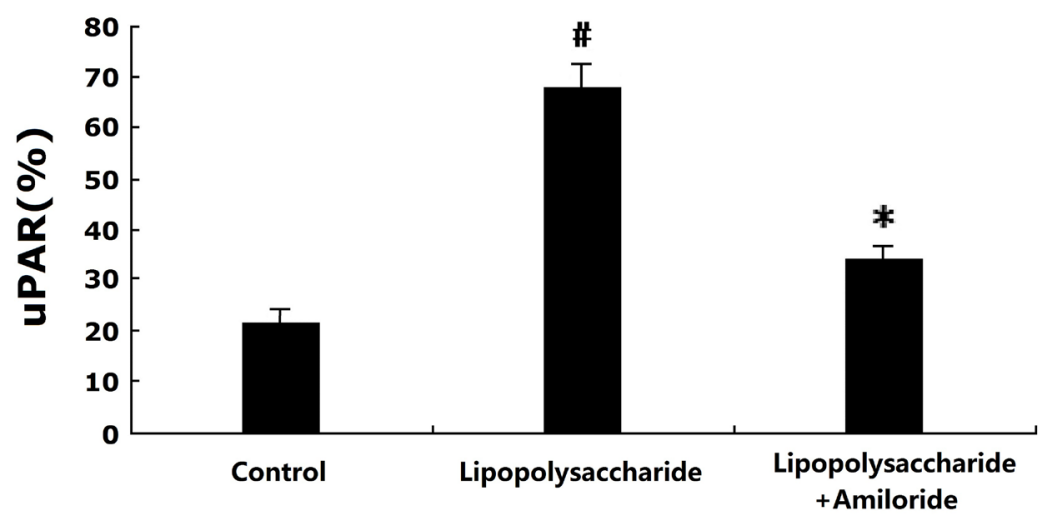

Figure 2. Effect of amiloride on uPAR protein expression in podocytes. ${ }^{*}$ Compared with the control group, the UPAR expression rate significantly increased in the lipopolysaccharide group, $\mathrm{P}<0.05$; *compared with the lipopolysaccharide group, the uPAR expression rate decreased somewhat in the lipopolysaccharide + amiloride group; these differences were statistically significant, $\mathrm{P}<0.05$. uPAR $=$ urokinase-type plasminogen activator receptor.

\section{Detecting the effect of uPAR on cell motility in podocytes with the transwell migration assay}

In the control, lipopolysaccharide, and lipopolysaccharide + amiloride groups, the average cell numbers in each field, representing cells that had undergone migration, were $162.38 \pm 23.71,328.14 \pm 35.12$, and $204.25 \pm 28.11$ respectively. The average cell number in the lipopolysaccharide group was significantly larger than those in the other two groups $(\mathrm{P}<$ 0.05) (Table 2 and Figure 3).

Table 2. Detection of podocyte motility changes using a transwell migration experiment.

\begin{tabular}{lccr}
\hline Group & Control & Lipopolysaccharide & Lipopolysaccharide + Amiloride \\
\hline Podocytes (means \pm SD) & $162.38 \pm 23.71$ & $328.14 \pm 35.12^{\#}$ & $204.25 \pm 28.11 *$ \\
\hline
\end{tabular}

\#Compared with the control group, $\mathrm{P}<0.05 ; *$ compared with the lipopolysaccharide group, $\mathrm{P}<0.05$.

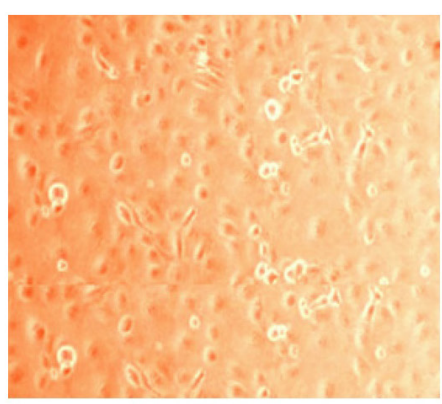

A

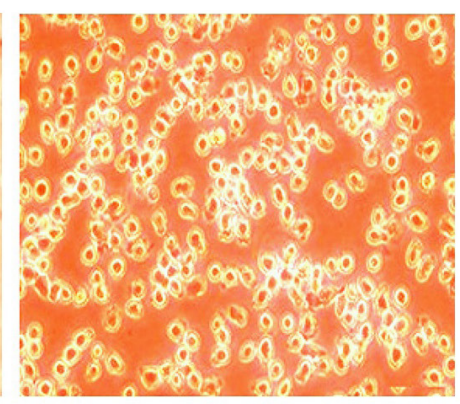

B

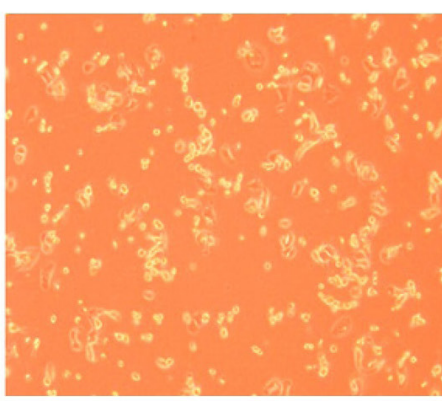

C

Figure 3. Detection of podocyte motility using a transwell migration experiment. A) Control group; B) lipopolysaccharide group; and C) lipopolysaccharide + amiloride group. It can be seen from the figure that cell migration occurred each group, particularly in the lipopolysaccharide group, $24 \mathrm{~h}$ after treatment. 


\section{Changes of proteinuria in the various groups in the model of mouse proteinuria induction by lipopolysaccharides}

Compared with the urine protein level in the control group over $24 \mathrm{~h}$, urine protein increased significantly in the lipopolysaccharide group $(\mathrm{P}<0.05)$. However, compared with the lipopolysaccharide group, the urine protein level decreased significantly in the lipopolysaccharide + amiloride group $(\mathrm{P}<0.05)$; there was no statistically significant difference observed following comparison between the lipopolysaccharide + amiloride and the control groups $(\mathrm{P}$ $>0.05$ ) (Table 3 and Figure 4).

Table 3. Urine protein levels over $24 \mathrm{~h}$ in mice of the different groups.

\begin{tabular}{lccc}
\hline Group & Control & Lipopolysaccharide & Lipopolysaccharide + Amiloride \\
\hline $\mathrm{N}$ & 10 & 10 & 10 \\
Level of urine protein over $24 \mathrm{~h}(\mathrm{mg} / \mathrm{mL})($ means $\pm \mathrm{SD})$ & $0.98 \pm 0.16$ & $4.17 \pm 0.23^{\#}$ & $1.73 \pm 0.22^{*}$
\end{tabular}

$\mathrm{N}=$ number of laboratory mice. ${ }^{*}$ Compared with the control group, $\mathrm{P}<0.05 ;{ }^{*}$ compared with the lipopolysaccharide group, $\mathrm{P}<0.05$.

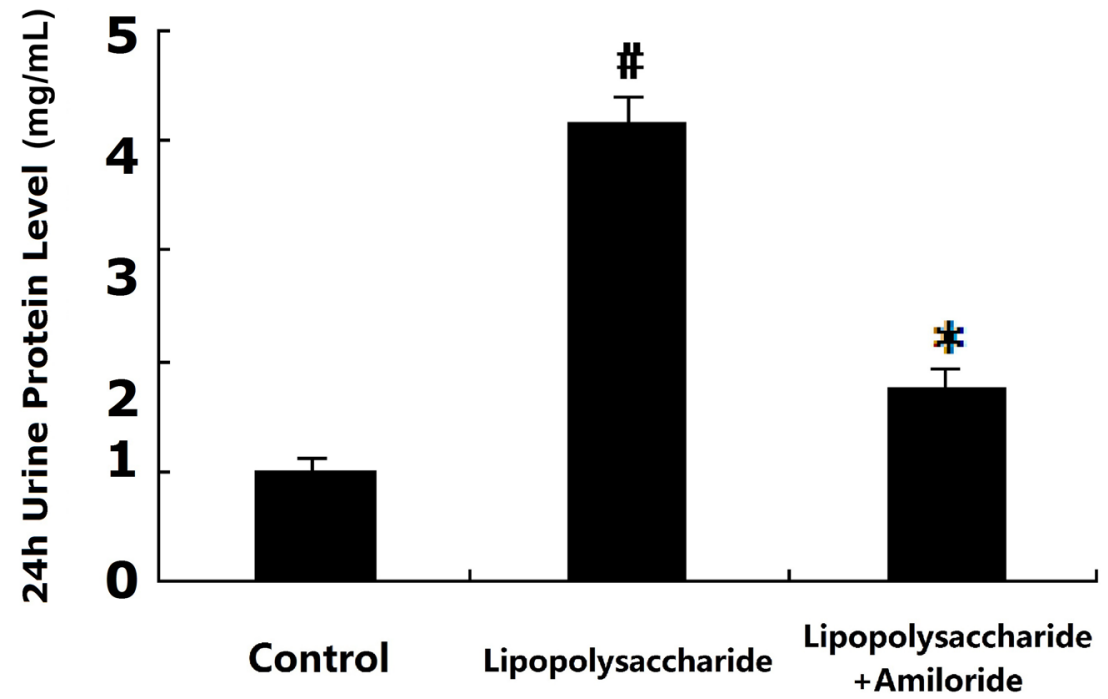

Figure 4. Comparison of urine protein levels over $24 \mathrm{~h}$ in mice of the different groups. ${ }^{\#}$ Compared with the control group, the urine protein levels significantly increased in the lipopolysaccharide group, $\mathrm{P}<0.05 ; *$ compared with the lipopolysaccharide group, the urine protein levels decreased somewhat in the lipopolysaccharide + amiloride group; this difference was statistically significant, $\mathrm{P}<0.05$.

\section{Amiloride might inhibit UPAR expression in podocytes in the mouse model of transient proteinuria induced by lipopolysaccharides}

In the mouse model of transient proteinuria induced by lipopolysaccharides, the podocyte-specific skelemin synaptopodin in the control group was found to be primarily expressed in glomerular podocytes, uPAR was widely present in renal tubules and glomeruli, and both 
were seldom observed simultaneously in glomerular podocytes. In comparison, in the lipopolysaccharide group, it was observed that uPAR expression had increased, the uPAR fluorescent brightness was high, and UPAR and synaptopodin appeared overlapping hybridization signals. In the lipopolysaccharide + amiloride group, it was observed that uPAR expression was downregulated significantly and that overlapping hybridization signals of synaptopodin was decreased on the glomerular podocytes (Figure 5).
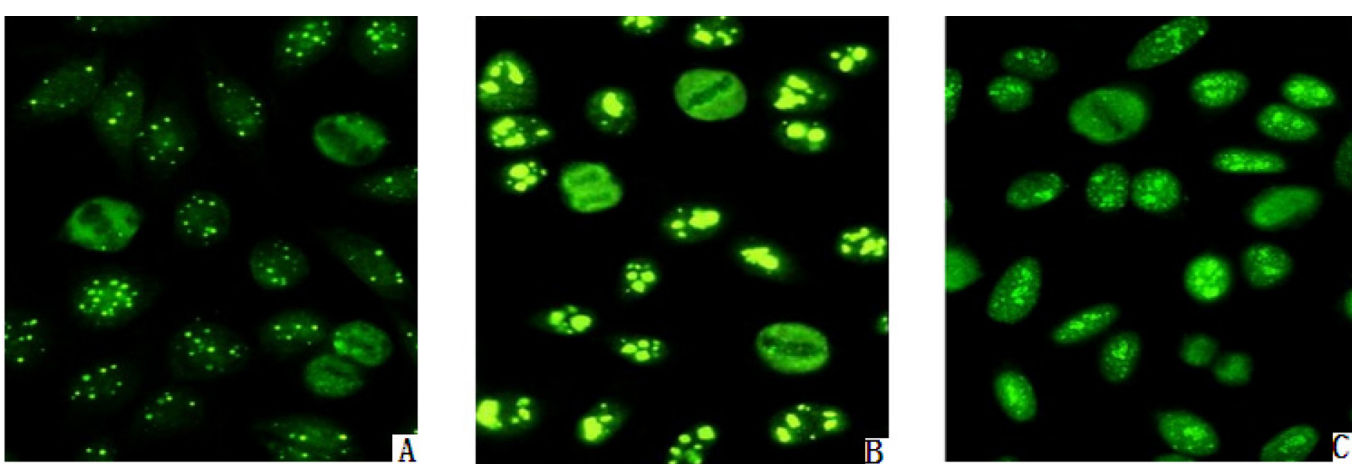

Figure 5. Expression and cellular localization of uPAR in mouse proteinuria models of the different groups. A) Control group; B) lipopolysaccharide group; and C) lipopolysaccharide + amiloride group. It can be seen from the figure that UPAR was least expressed in the control group as demonstrated by the low fluorescence intensity, most highly expressed in the lipopolysaccharide group, and expressed to a somewhat lesser degree in the lipopolysaccharide + amiloride group. $\mathrm{UPAR}=$ urokinase-type plasminogen activator receptor.

\section{DISCUSSION}

Proteinuria is not only a sign of renal injury and an index of the efficacy of clinical treatment, but is also an important risk factor in the process whereby glomerular disease progresses to renal tubule injury and interstitial fibrosis and finally to chronic kidney disease (CKD) (Zhang et al., 2013). Podocytes and proteinuria are involved in almost all glomerular diseases. As the final barrier of the glomerulus filtration barriers, podocytes have become the critical cells for current research on proteinuria. Enhanced mobility and fusion of podocytic processes damage the glomerulus filtration barriers thus leading to the occurrence of proteinuria. Therefore, podocyte damage is directly associated with the occurrence of proteinuria (Liu et al., 2013; Worthmann et al., 2013). To date, substantial progress has been made in the research on the molecular structure of podocytes and the understanding of their interactions. In addition, further in-depth research on the damage to podocytes and the changes of their molecular structure that occur during proteinuria has been performed. Under normal physiological status, the maturely differentiated podocytes are terminal cells without division and proliferation capacity that exist in a relatively stationary state. In contrast, damaged podocytes are hyperdynamic cells that bear similarity to tumor cells (Merscher-Gomez et al., 2013; Yu et al., 2013b). In vitro experiments have shown that these cells exhibit enhanced migration and invasion capacities; i.e. their mobility is enhanced, while in vivo experiments have demonstrated changes in the dynamic system of the podocytic processes and podocytic process fusion (Mao et al., 2013; Shen et al., 2013).

As a transmembrane protein, which regulates cell activities, uPAR plays an important 
role in inflammation, tumor infiltration, and metastasis (Trimarchi, 2013). Previous research has found that uPAR is an important molecular protein in regulating tumor cells and their infiltration and metastasis of the surrounding tissues indicating that uPAR plays an important role in regulating tumor cell motility (Lugano et al., 2012; Yeh et al., 2013). Studies have also shown that the expression level of UPAR rises and a large amount of proteinuria appears in models of animals with nephrotoxic nephritis, thus giving rise to the reconstruction of renal tissues and glomerular sclerosis (Boucher et al., 2012; Yasuno et al., 2013). The resultant damaged podocytes exhibit features similar to those of tumor cells, and their motility is also strengthened considerably (Brähler et al., 2012). Recent research has found that increased UPAR expression can enhance the activities of podocytes and the generation of proteinuria in diseased human glomeruli, nephropathy animal models, and in proteinuria animal and cell models induced by lipopolysaccharide. To study these issues, researchers have utilized gene knock-out and transgenic animals. It was found that the proteinuria of the injured mouse following lipopolysaccharide induction decreased significantly upon knock-out of the $u P A R$ gene, whereas it increased significantly after the $U P A R$ cDNA was re-introduced, indicating that $U P A R$ plays a unique role in the pathogenic mechanism of proteinuria (Meyer-Schwesinger et al., 2011; Xiao et al., 2011). Research has also shown that damaged podocytes are able to increase the motility of normal podocytes and can cause foot process fusion and the occurrence of proteinuria by up-regulating the expression of uPAR (Patrakka and Tryggvason, 2009).

As a classic diuretic, amiloride primarily acts on distal renal tubules and blocks sodiumpotassium exchange to promote the excretion of sodium and chlorine and reduce the secretion of potassium and hydrogen ions, thus achieving diuresis (Sun et al., 2012). It has been reported that amiloride might inhibit uPAR RNA and protein synthesis in cloned tumor cell strains (Haishima et al., 2012; Shkreli et al., 2012). Based on the previously discussed research, these findings suggest the question of whether amiloride is able to reduce the motility of podocytes thus stabilizing them and lowering the effects of proteinurea on the damaged podocytes.

In this study, an in vitro model of podocyte damage induced by lipopolysaccharides was established and amiloride was used for interventional treatment. We observed that the expression of UPAR in the podocytes in the lipopolysaccharide group was significantly higher than that in the control group and that the expression of UPAR decreased significantly after interventional amiloride treatment, indicating that amiloride was able to inhibit the expression of the uPAR protein in podocytes. To detect the changes in the motility of podocytes in the various treatment groups, a transwell migration experiment and a scraping experiment were used. The motility of the damaged podocytes increased significantly in the lipopolysaccharide group and the motility decreased after intervention with amiloride, which contributed to the stabilization of the podocytes and to damage repair. Thus, at the level of podocytes in an in vitro experiment, amiloride was able to inhibit the UPAR expression of damaged podocytes, decrease the motility and migration capacity of podocytes, decrease their activities, and to stabilize them and return them partially or completely to their normal stationary state. In the in vivo experiment, we established a mouse model of transient proteinuria induced by lipopolysaccharide. We used amiloride to intervene in the establishment of the animal model and detected the proteinuria over $24 \mathrm{~h}$. We observed that amiloride was able to significantly decrease the occurrence of proteinuria.

Based on the above results, we concluded that amiloride is able to reduce the motility of podocytes and stabilize them, and thus lower the overall proteinuria by inhibiting the expression of uPAR in the podocytes. Amiloride thus can be considered a new medicine for lowering proteinuria in podocyte-targeted cell treatments. 


\section{Conflicts of interest}

The authors declare no conflict of interest.

\section{REFERENCES}

Abe Y, Sakairi T, Beeson C and Kopp JB (2013). TGF-beta1 stimulates mitochondrial oxidative phosphorylation and generation of reactive oxygen species in cultured mouse podocytes, mediated in part by the mTOR pathway. Am. J. Physiol. Renal. Physiol. 305: F1477-1490.

Boucher I, Yu W, Beaudry S, Negoro H, et al. (2012). Ga12 activation in podocytes leads to cumulative changes in glomerular collagen expression, proteinuria and glomerulosclerosis. Lab. Invest. 92: 662-675.

Brähler S, Ising C, Hagmann H, Rasmus M, et al. (2012). Intrinsic proinflammatory signaling in podocytes contributes to podocyte damage and prolonged proteinuria. Am. J. Physiol. Renal. Physiol. 303: F1473-1485.

Haishima A, Murakami M, Ikeda T, Inoue K, et al. (2012). Detection of Bcl-2 mRNA and its product in the glomerular podocytes of the normal rat kidney. Exp. Toxicol. Pathol. 64: 633-637.

Hasegawa K, Wakino S, Simic P, Sakamaki Y, et al. (2013). Renal tubular Sirt1 attenuates diabetic albuminuria by epigenetically suppressing Claudin-1 overexpression in podocytes. Nat. Med. 19: 1496-1504.

Ilatovskaya DV, Palygin O, Levchenko V and Staruschenko A (2013). Pharmacological characterization of the P2 receptors profile in the podocytes of the freshly isolated rat glomeruli. Am. J. Physiol. Cell Physiol. 305: 1050-1059.

Lasagni L and Romagnani P (2013). Basic research: Podocyte progenitors and ectopic podocytes. Nat. Rev. Nephrol. 9: 715-716.

Li D, Wang N, Zhang L, Hanyu Z, et al. (2013). Mesenchymal stem cells protect podocytes from apoptosis induced by high glucose via secretion of epithelial growth factor. Stem Cell Res. Ther. 4: 103.

Liu Y, Liang W, Yang Q, Ren Z, et al. (2013). IQGAP1 mediates angiotensin II-induced apoptosis of podocytes via the ERK1/2 MAPK signaling pathway. Am. J. Nephrol. 38: 430-444.

Lugano R, Peña E, Badimon L and Padró T (2012). Aggregated low-density lipoprotein induce impairment of the cytoskeleton dynamics through urokinase-type plasminogen activator/urokinase-type plasminogen activator receptor in human vascular smooth muscle cell. J. Thromb. Haemost. 10: 2158-2167.

Mao J, Wang D, Mataleena P, He B, et al. (2013). Myole impairment results in actin reorganization, podocyte dysfunction, and proteinuria in zebrafish and cultured podocytes. PLoS One 8: e72750.

Merscher-Gomez S, Guzman J, Pedigo CE, Lehto M, et al. (2013). Cyclodextrin protects podocytes in diabetic kidney disease. Diabetes 62: 3817-3827.

Meyer-Schwesinger C, Meyer TN, Sievert H, Hoxha E, et al. (2011). Ubiquitin C-terminal hydrolase-11 activity induces polyubiquitin accumulation in podocytes and increases proteinuria in rat membranous nephropathy. Am. J. Pathol. 178: 2044-2057.

Müller-Krebs S, Weber L, Tsobaneli J, Kihm LP, et al. (2013). Cellular effects of everolimus and sirolimus on podocytes. PLoS One 8: e80340.

Patrakka J and Tryggvason K (2009). New insights into the role of podocytes in proteinuria. Nat. Rev. Nephrol. 5: 463-468.

Reiser J (2013). Akt2 relaxes podocytes in chronic kidney disease. Nat. Med. 19: 1212-1213.

Sanchez-Niño MD, Poveda J, Sanz AB, Mezzano S, et al. (2013). Fn14 in podocytes and proteinuric kidney disease. Biochim. Biophys. Acta 1832: 2232-2243.

Shen L, Lu G, Dong N, Ma Z, et al. (2013). Simvastatin increases ADAMTS13 expression in podocytes. Thromb. Res. 132: 94-99.

Shi S, Yu L, Zhang T, Qi H, et al. (2013). Smad2-dependent downregulation of miR-30 is required for TGF-beta-induced apoptosis in podocytes. PLoS One 8: e75572.

Shkreli M, Sarin KY, Pech MF, Papeta N, et al. (2012). Reversible cell-cycle entry in adult kidney podocytes through regulated control of telomerase and Wnt signaling. Nat. Med. 18: 111-119.

Sun D, Zhao X and Meng L (2012). Relationship between urinary podocytes and kidney diseases. Ren. Fail. 34: 403-407.

Trimarchi H (2013). Primary focal and segmental glomerulosclerosis and soluble factor urokinase-type plasminogen activator receptor. World J. Nephrol. 2: 103-110.

Wei C, Moller CC, Altintas MM, Li J, et al. (2008). Modification of kidney barrier function by the urokinase receptor. Nat. Med. 14: 55-63.

Worthmann K, Leitges M, Teng B, Sestu M, et al. (2013). Def-6, a novel regulator of small GTPases in podocytes, acts downstream of atypical protein kinase C (aPKC) $\lambda /$. Am. J. Pathol. 183: 1945-1959. 
Xiao Z, He L, Takemoto M, Jalanko H, et al. (2011). Glomerular podocytes express type 1 adenylate cyclase: inactivation results in susceptibility to proteinuria. Nephron Exp. Nephrol. 118: e39-48.

Yasuno K, Kamiie J and Shirota K (2013). Analysis of ultrastructural glomerular basement membrane lesions and podocytes associated with proteinuria and sclerosis in Osborne-Mendel rats with progressive glomerulonephropathy. J. Vet. Sci. 14: 223-226.

Yeh YH, Wang PH, Lin LY, Tee YT, et al. (2013). Significantly increased concentration of soluble urokinase-type plasminogen activator receptor in the blood of patients with pelvic inflammatory disease. Clin. Chim. Acta 415: 138-144.

Yu H, Suleiman H, Kim AH, Miner JH, et al. (2013a). Rac1 activation in podocytes induces rapid foot process effacement and proteinuria. Mol. Cell Biol. 33: 4755-4764.

Yu SY, Qi R and Zhao H (2013b). Losartan reverses glomerular podocytes injury induced by AngII via stabilizing the expression of GLUT1. Mol. Biol. Rep. 40: 6295-6301.

Zhang B, Xie S, Shi W and Yang Y (2012). Amiloride off-target effect inhibits podocyte urokinase receptor expression and reduces proteinuria. Nephrol. Dial. Transplant. 27: 1746-1755.

Zhang X, Qu X, Sun YB, Caruana G, et al. (2013). Resolvin D1 protects podocytes in adriamycin-induced nephropathy through modulation of 14-3-3 $\beta$ acetylation. PLoS One 8: e67471. 